\title{
Revising the exclusivity of Pemberton's sign: A case report
}

\author{
Philip W. Tipton, Jeremy J. Blanchard, Will P. Guider, Jessica J. Keel, Allison D. Locke, \\ Franchesca N. Robichaud, Adam Price, William L. Joyner, John K. Smith*
}

Department of Internal Medicine, J.H. Quillen College of Medicine East Tennessee State University, Johnson City, TN, USA

\section{Email address:}

ptipton3@gmail.com (P. W. Tipton), blanchard@goldmail.etsu.edu (J. J. Blanchard), guider@goldmail.etsu.edu (W. P. Guider), keeljj@goldmail.etsu.edu (J. J. Keel), zads46@goldmail.etsu.edu (A. D. Locke), robichaud@goldmail.etsu.edu (F. N. Robichaud), priceab@goldmail.etsu.edu (A. Price), JOYNERW@mail.etsu.edu (W. L. Joyner), SMITHJ@mail.etsu.edu (J. K. Smith)

\section{To cite this article:}

Philip W. Tipton, Jeremy J. Blanchard, Will P. Guider, Jessica J. Keel, Allison D. Locke, Franchesca N. Robichaud, Adam Price, William L. Joyner, John K. Smith. Revising the Exclusivity of Pemberton's Sign: A Case Report. American Journal of Internal Medicine. Vol. 2, No. 3, 2014, pp. 41-43. doi: 10.11648/j.ajim.20140203.11

\begin{abstract}
The Pemberton maneuver is a tool that may be utilized during the physical examination to demonstrate the presence of latent pressure in the thoracic inlet [1]. The maneuver is achieved by having the patient elevate both arms until they touch the sides of the face. A positive sign is marked by the presence of facial congestion and cyanosis, as well as respiratory distress after approximately one minute [2]. The sign is most commonly present in patients with substernal goiters where the goiter "corks off" the thoracic inlet [3]. We describe a 67-year-old Caucasian male with a positive Pemberton's sign due to compression of the thoracic inlet by enlarged mediastinal nodes. The sign was amplified by the presence of a restrictive cardiomyopathy and constrictive pericarditis due to amyloidosis. We propose that a positive Pemberton's sign should not be used exclusively to evaluate the probability of a substernal goiter, but rather to assess the potential for vascular compression due to pathological changes in the anatomical environment of the thoracic inlet.
\end{abstract}

Keywords: Permberton's Sign, Lymphadenopathy, Restrictive Cardiomyopathy, Constrictive Pericarditis, Geriatrics, Amyloidosis

\section{Introduction}

Today's practicing physician commonly encounters congestive heart failure. While many characteristic features of volume overload are easily appreciated, a more focused and thoughtful approach to the physical examination is often needed to provide evidence capable of suggesting etiology. Obstruction is one possible etiology when dealing with appreciable volume overload. Moreover, localization of obstructions can prove quite valuable in the pursuit of diagnoses and subsequent treatment.

The Pemberton maneuver is a physical examination tool used to demonstrate the presence of latent pressure in the thoracic inlet [1]. The maneuver is achieved by having the patient elevate both arms until they touch the sides of the face. A positive sign is marked by the presence of facial congestion and cyanosis, as well as respiratory distress after approximately one minute [2]. Although the sign is most commonly described in patients with substernal goiters where the goiter "corks off" the thoracic inlet [3], the maneuver is potentially useful in any patient with adenopathy, tumor, or fibrosis involving the mediastinum.

Diagnosis of restrictive cardiomyopathy is often difficult because much of the symptomatology is also commonly associated with constrictive pericarditis. Constrictive pericarditis is a dense fibrous thickening of the pericardium that causes chronic cardiac compression, resulting in restricted diastolic filling of the heart. This is frequently observed in the form of venous engorgement and diminished cardiac output [4]. Restrictive cardiomyopathy typically results from increased stiffness of the myocardium that causes pressure within the ventricle to rise precipitously with only small increases in volume. This can occur in one or both ventricles, thereby allowing clinical presentation to include signs of right or left heart failure [5]. There are multiple causes of both restrictive cardiomyopathy and constrictive pericarditis. One such cause is accumulation of the protein amyloid [6,7], which has also been linked to some rare cases of mediastinal lymphadenopathy [8]. 


\section{Case Presentation}

A 67-year-old Caucasian male with congestive heart failure and chronic atrial fibrillation presented to the emergency department with complaints of increasing dyspnea, orthopnea, paroxysmal nocturnal dyspnea, weight gain, and dependent edema unresponsive to increasing doses of Lasix. His past medical history was positive for hypertension, chronic obstructive pulmonary disease, and liver cirrhosis due to excess alcohol consumption. His family history was positive for atherosclerotic heart disease, and cancer of the lung and breast.

Upon physical examination, he appeared chronically ill and was in considerable respiratory distress. His blood pressure was $107 / 69 \mathrm{mmHg}$, pulse 88 and irregularly irregular, respiratory rate 18 , and temperature $97.5^{\circ} \mathrm{F}$. Pertinent physical examination findings included a paradoxical pulse of $20 \mathrm{mmHg}$, jugular venous distention, positive Kussmaul's and Pemberton's signs, bilateral crackles in the middle and lower lung fields, bibasilar dullness, and pitting edema to the level of the sternal notch. His heart was enlarged to percussion with distant heart sounds and an audible pericardial knock. Several engorged veins were present in the right infraclavicular region.

Blood studies were notable for mild pancytopenia and an elevated N-terminal prohormone of $\operatorname{BNP}(3,450 \mathrm{pg} / \mathrm{mL}$; reference range $0-124 \mathrm{pg} / \mathrm{mL}$ ). Chest $\mathrm{X}$-ray showed the heart size to be at the upper limits of normal while an electrocardiogram (ECG) revealed low voltage and atrial fibrillation. An echocardiogram was unremarkable showing no evidence of acute coronary syndrome, valvular pathology, or cardiac tamponade. Computerized tomography (CT) of the chest was obtained as the patient's symptoms persisted. Findings included a dilated right ventricle, pericardial thickening, as well as pericardial and pleural effusions (Fig. 1). More superior CT cuts reveal moderate diffuse mediastinal adenopathy (Fig. 2).

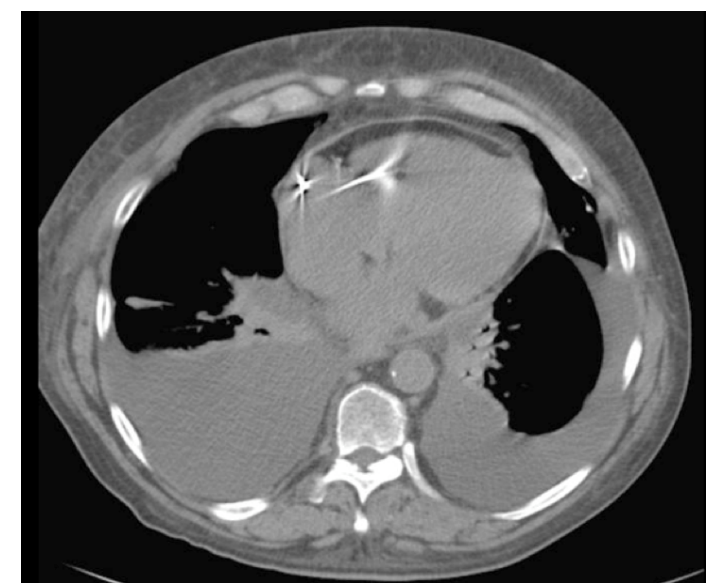

Figure 1. A CT scan of the thorax showing pericardial and pleural effusions as well as thickening of the pericardium.

Cardiac catheterization was recommended for further evaluation to assess the severity of his condition and to confirm CT findings suggestive of pericarditis. The catheterization showed equalization of right ventricular end diastolic pressure (RVEDP) and left ventricular end diastolic pressure (LVEDP) with a dip and plateau configuration (square root sign of restrictive cardiomyopathy) of pressures in both ventricles. Furthermore, equalized filling pressures in all four chambers (a sign of constrictive pericarditis) were observed. Pulmonary artery pressure was 47/26 mmHg with a mean of $34 \mathrm{mmHg}$. The mean pulmonary capillary wedge pressure was $20 \mathrm{mmHg}$ and the pulmonary vascular resistance was 2.65 Wood units. The cardiac output was 4.91 $\mathrm{L} / \mathrm{min}$. Additional diagnostics included a right ventricular endomyocardial biopsy, which revealed the presence of amyloid.

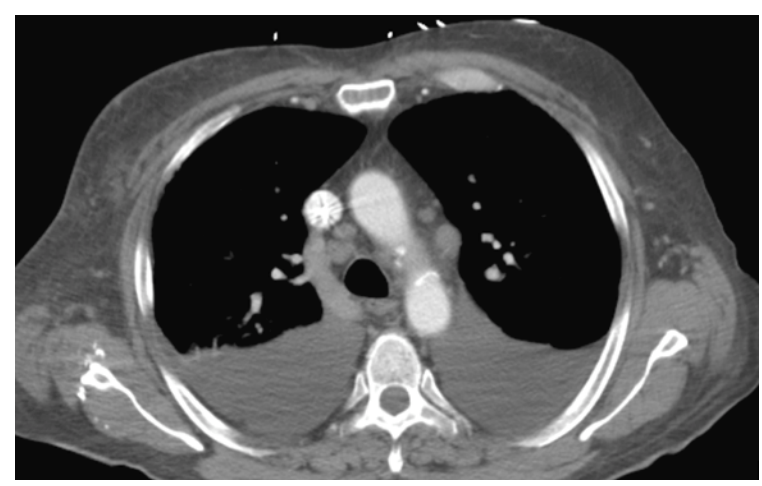

Figure 2. CT scan at the level of the aortic arch showing diffuse mediastinal lymphadenopathy and bilateral pleural effusions.

\section{Discussion}

Previous studies have reported amyloidosis as a cause of not only restrictive cardiomyopathy [7], but also constrictive pericarditis [6] and mediastinal lymphadenopathy [8]. We present an unusual case of amyloidosis in which all three of these pathologies are present. The exact manner in which each component of this amalgam of pathologies contributed to our patient's positive Pemberton's sign is difficult to discern. Thoracic outlet syndrome, also known as superior vena cava (SVC) syndrome, has been observed as a result of diffuse mediastinal lymphadenopathy of various pathologies such as cystic fibrosis [9] and Castleman's disease [10]. Park et al. reported enlarged cervical lymph nodes associated with hemophagocytic lymphohistiocytosis as the cause of internal jugular vein compression, which presented clinically similar to SVC syndrome [11]. In our patient, it is likely that the maneuver caused his mediastinal nodes to "cork off" his thoracic inlet. Moreover, his cardiac conditions, by further impairing venous return, amplified the development of facial cyanosis, facial edema, and respiratory distress. Furthermore, it is of interest that our patient had engorged veins in the right infraclavicular region of his chest, suggesting that these perforating veins were chronically obstructed just distal to their entry into the right internal thoracic vein, possibly by mediastinal nodes (Fig. 3).

Several findings in this case are common to both constrictive pericarditis and restrictive cardiomyopathy. 
Among these are Kussmaul's sign, pulsus paradoxus, peripheral edema, mild cardiomegaly, and low-voltage ECG. Some symptoms; however, are typically associated with one or the other. The presence of a pericardial knock is found in approximately $50 \%$ of patients with constrictive pericarditis and not typically associated with restrictive cardiomyopathy [12]. Conversely, elevated BNP levels (released in response to ventricular dysfunction and wall stretch) are characteristic of restrictive cardiomyopathy while levels typically remain normal in constrictive pericarditis [13]. Cardiac catheterization findings were also unsuccessful in truly differentiating between these etiologies. While the square root sign is typical of restrictive cardiomyopathy, four-chamber filling pressure equalization is considered a major diagnostic criterion of constrictive pericarditis [14]. Moreover, the addition of a positive Pemberton's sign typically eludes both of the previously mentioned cardiac pathologies. Taken together, this unique combination of physical examination findings and diagnostic test results support the claim that multiple pathologies are at work. Cases such as this serve as a reminder of the value of bedside diagnosis.

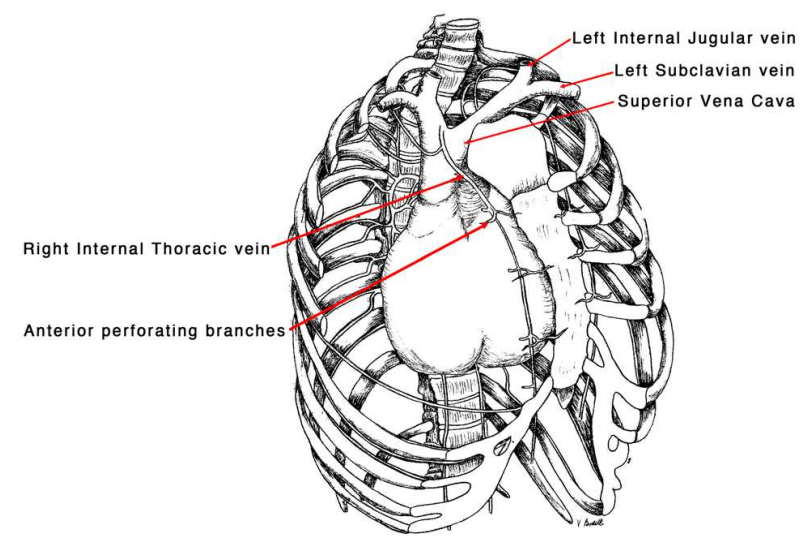

Figure 3. Illustration of the thoracic venous circulation.

\section{Conclusion}

The Pemberton maneuver can be useful in detecting latent thoracic inlet obstruction due to a plethora of causes. Preexisting cardiac conditions that impair venous return may serve to amplify the findings of facial cyanosis, facial edema, and respiratory distress that characterize a positive Pemberton's sign.

\section{Acknowledgements}

Thank you to Valerie Bodell, who is the artist of figure 3 (thoracic cavity).

\section{Nomenclature}

BNP (brain natriuretic peptide), ECG (electrocardiogram), (CT) computerized tomography, RVEDP (right ventricular end diastolic pressure), LVEDP (left ventricular end diastolic pressure), SVC (superior vena cava).

\section{References}

[1] Wallace, C. and Siminoski, K. The Pemberton sign. Ann Intern Med 1996, 125:568-569.

[2] Pemberton, H.S. Sign of submerged goitre. Lancet 1946, 251:509.

[3] Basaria, S. and Salvatori, R. Pemberton's sign. N Engl J Med 2004, 350:1338.

[4] Mehta, A., Mehta, M., and Jain, A.C. Constrictive pericarditis. Clin Cardiol 1999, 22:334-344.

[5] Kushwaha, S.S., Fallon, J.T., and Fuster, V. Restrictive cardiomyopathy. N Engl J Med 1997, 336:267-276.

[6] Sing, V., Fishman, J.E., and Alfonso, C.E. Primary systemic amyloidosis presenting as constrictive pericardidits. Cardiology 2011, 118:251-255.

[7] Guan, J., Mishra, S., Falk, R.H., and Liao, R. Current perspectives on cardiac amyloidosis. Am J Physiol Heart Circ Physiol 2012, 302:544-552.

[8] Takeshita, K., Yamada, S., and Sato, N. An unusual case of mediastinal lymphadenopathy caused by amyloidosis. Intern Med 2000, 39:839-842.

[9] Chow, B.J., McKim, D.A., Shennib, H. et al. Superior vena cava obstruction secondary to mediastinal lymphadenopathy in a patient with cystic fibrosis. Chest 1997, 112:1438-1441.

[10] Tekinbas, C., Erol, M.M., Ozsu, S. et al. Giant mass due to Castleman's disease causing superior vena cava syndrome. Thorac Cardiovasc Surg 2008, 56:303-305.

[11] Park, M., Choi, J.W., Park, H.J. et al. Hemophagocytic lymphohistiocytosis can mimic the superior vena cava syndrome. J Pediatr Hematol Oncol 2012, 34:152-154.

[12] Ling, L.H., Oh, J.K., Schaff, H.V. et al. Constrictive pericarditis in the modern era: evolving clinical spectrum and impact on outcome after pericardectomy. Circulation 1999, 100:1380-1386.

[13] Leya, F.S., Arab, D., Joyal, D. et al. The efficacy of brain natriuretic peptide levels in differentiating constrictive pericarditis from restrictive cardiomyopathy. J Am Coll Cardiol 2005, 45:1900-1902.

[14] Hirota, Y., Kohriyama, T., Hayashi, T. et al. Idiopathic restrictive cardiomyopathy: differences of left ventricular relaxation and diastolic wave forms from constrictive pericarditis. Am J Cardiol 1983, 52:421-423. 